Bull. Chem. Soc. Ethiop. 2021, 35(2), 337-350.

(c) 2021 Chemical Society of Ethiopia and The Authors

ISSN 1011-3924

DOI: https://dx.doi.org/10.4314/bcse.v35i2.9

Printed in Ethiopia

Online ISSN 1726-801X

\title{
CO-ORDINATION BEHAVIOUR OF HYDRAZINE AND ISOMERS OF ACETOXY BENZOIC ACIDS WITH TRANSITION METAL IONS
}

\author{
E. Helen Pricilla Bai ${ }^{1}$ and S. Vairam ${ }^{2 *}$ \\ ${ }^{1}$ Department of Chemistry, Park College of Engineering and Technology, Coimbatore-641659, \\ India \\ ${ }^{2}$ Department of Chemistry, KPR Institute of Engineering and Technology, Coimbatore-641407, \\ India
}

(Received December 21, 2020; Revised October 4, 2021; Accepted October 4, 2021)

\begin{abstract}
New hydrazine complexes of some divalent transition metal ions of formulae, $\left[\mathrm{M}\left\{(2-\mathrm{ab})_{2}\left(\mathrm{~N}_{2} \mathrm{H}_{4}\right)\right\}\right] \cdot 3 \mathrm{H}_{2} \mathrm{O},\left[\mathrm{M}\left\{(3-\mathrm{ab})_{2}\left(\mathrm{~N}_{2} \mathrm{H}_{4}\right)\right\}\right] \cdot 3 \mathrm{H}_{2} \mathrm{O}$ and $\left[\mathrm{M}\left\{(4-\mathrm{ab})_{2}\left(\mathrm{~N}_{2} \mathrm{H}_{4}\right)_{2}\right\}\right] \cdot 2 \mathrm{H}_{2} \mathrm{O}$ where $\mathrm{M}=\mathrm{Co}, \mathrm{Ni}, \mathrm{Zn}$ and $\mathrm{Cd} ; 2$-abH $=2$-acetoxy benzoic acid, 3 -abH $=3$-acetoxy benzoic acid and 4-abH = 4-acetoxy benzoic acid were prepared using their respective metal nitrates and hydrazine hydrate at $\mathrm{pH} 6$ and 5 , respectively, and characterized by elemental analysis, IR and UV-reflectance spectroscopic techniques, thermo analytical technique, powder XRD, SEM-EDX and magnetic susceptibility measurements. All the transition metal complexes were sparingly soluble in water. The IR spectra of complexes showed N-N absorptions of hydrazine in the range of $964-988 \mathrm{~cm}^{-1}$ substantiating the bidentate bridging coordination of hydrazine. They showed endothermic decomposition in the range of $67-100{ }^{\circ} \mathrm{C}$, exothermic dehydrazination in the range, $210-281{ }^{\circ} \mathrm{C}$ and an oxidative decomposition between 350 and $490^{\circ} \mathrm{C}$ to form their respective metal oxides. The electronic spectral and magnetic susceptibility data could substantiate the distorted octahedral geometry of nickel and cobalt complexes and the XRD pattern indicates the existence of isomorphism. Crystals of $\left[\mathrm{Cd}\left(\mathrm{H}_{2} \mathrm{O}\right)_{3}\left(\left(\mathrm{C}_{6} \mathrm{H}_{4}(4-\mathrm{OH})(\mathrm{COO})\right)_{2}\right] \cdot \mathrm{H}_{2} \mathrm{O}\right.$ obtained as by-product, was also characterized using single crystal XRD, IR and thermal studies.
\end{abstract}

KEY WORDS: Acetoxy benzoic acids, Hydrazine, Thermal analysis, Single crystal XRD

\section{INTRODUCTION}

The isomers of acetoxy benzoic acids $(\mathrm{abH})$ are capable ligands for the formation of complexes owing to the presence of $\mathrm{COOH}$ and $\mathrm{CH}_{3} \mathrm{COO}$ - groups attached with benzene ring. The 2-abH isomer, commonly called as aspirin, is a most frequently used antipyretic and 4-abH has been used in polymer synthesis. Literature survey indicates that these acids, especially 2 and 3-abH can form metal complexes having certain therapeutic effects such as due to the complexation of the metallic ion $\left(\mathrm{Cu}^{2+}, \mathrm{Zn}^{2+}, \mathrm{Fe}^{2+}, \mathrm{Mg}^{2+}\right.$, etc.) essential for a certain biochemical process. Obaleye et al. reported the anti-bacterial activity of cobalt, nickel and iron complexes of aspirin. They found that 2-abH acted as bidentate chelate coordinating through carbonyl oxygen of carboxyl and ester groups and the metal complexes had greater antibacterial activity than that of ligand [1]. Ashraf et al. synthesized 2-acetoxy-4-(picolinamido)benzoic acid, using 2-abH and suggested that it was a promising anti-cancer drugs [2]. Study on modulation of biological properties of aspirin by formation of bio-organometallic derivative has been reported by Ott et al. [3]. In another report, biological activities of transition metal complexes based on 2-abH have been studied by Rubner et al. In this work, it was shown that there was increased inhibitory action against various tumour cells [4]. $\mathrm{Cu}$ (II) complexes of halogen substituted 2-abH have been prepared and their thermal behavior have been studied by Tarulli et al. [5]. Further, synthesis, spectroscopic and biological assessment of alkaline earth metal aspirinate complexes have been reported by Refat et al. [6]. Biological studies of novel mixed ligand $\mathrm{Ag}(\mathrm{I})$ complexes with triphenylphosphine and alicylic acid were also reported by Poyraz et al. [7]. Ogodo et al.

*Corresponding author. E-mail: drsvairam@rediffmail.com

This work is licensed under the Creative Commons Attribution 4.0 International License 
have reported green synthesis of $\mathrm{Cu}$ (II) complexes using salicylic acid and 2-abH [8]. Antimicrobial activity of organaotin complexes of aspirin has been reported by Kaur et al. [9]. Synthesis and characterization of tris-tolylbismuth(V) di-carboxylato complexes and their toxicity towards Leishmania promastigotes and human fibroblast cells have been studied by Ong et al. [10]. Islam et al. have synthesized triphenylbismuth(V) dicarboxylate complexes by the reaction of 2 and 3-acetoxy benzoic acids with $\mathrm{Ph}_{3} \mathrm{BiCl}_{2}$ [11]. Antitumor-active cobaltalkyne complexes derived from acetylsalicylic acid have been studied by Ott et al. [12]. Using 2-abH, synthesis of ruthenium(II) arene NSAID complexes is also finding place in the literature [13]. Aziz et al. studied the synthesis and properties the complexes from a mixture of aspirin (acetylsalicylic acid), paracetamol and methyldopa with divalent manganese, iron, cobalt, nickel and copper [14].

Despite so many reports on 2 and 3-isomers of acetoxy benzoic acids, no report on metal complexes using 4-acetoxybenzoic acids with metals is found in the literature. The main focus on the use of 4-isomer had been in the synthesis of polymers. Studies have been carried out on thermodynamic parameters of co-polycondensation of 4-abH and 6-acetoxy-2-naphthoic acids [15], kinetics of the polymerization of 4-abH and 6-acetoxy-2-naphthoic acids [16], vapour pressures, enthalpies and entropies of sublimation of para substituted benzoic acids [17], rheological and thermal properties of blended polyester [18] and synthesis of liquid crystalline polymers [19]. Pharmaceutical application of 2-abH was carried out by Chipley et al. [20].

In our laboratory, investigation on metal complexes of substituted aromatic carboxylic acids using hydrazine as co-ligand, with the aim of obtaining information about the coordination and thermal reactivity of the hydrazine of such complexes is being carried out. Hydrazine complexes of the first transition metal ions with a variety of carboxylic acids have been reported in the literature. These include simple and substituted aromatic mono [21-25], di [26-27], tricarboxylic [28] and tetracarboxylic acids. [29]. Extension of our idea of using new carboxylic acids made us work with acetoxy benzoic acids. A report was made by us with 3 and 4-abH using hydrazine [30]. Finding the fact that there is no other report on these acids using hydrazine, an attempt was made to synthesize hydrazine complexes of transition metal carboxylates. In this work, synthesis of hydrazine complexes of cobalt, nickel, cadmium and zinc acetoxy benzoates. Further, structural study of cadmium $p$-hydroxybenzoate prepared by an indirect method using 4 -abH is also presented.

\section{EXPERIMENTAL}

The solvents were distilled prior to use and double distilled water was used for the preparation and chemical analyses. The chemicals used were of AR grade. In all the reactions, $99-100 \%$ pure hydrazine hydrate was used as received.

Preparation of $\left[\mathrm{M}\left\{(2-a b)_{2}\left(\mathrm{~N}_{2} \mathrm{H}_{4}\right)\right\}\right] .3 \mathrm{H}_{2} \mathrm{O}$ and $\left[\mathrm{M}\left\{(3-\mathrm{ab})_{2}\left(\mathrm{~N}_{2} \mathrm{H}_{4}\right)\right\}\right] .3 \mathrm{H}_{2} \mathrm{O}$ where $\mathrm{M}=\mathrm{Co}, \mathrm{Ni}, \mathrm{Zn}$ and $C d$

The preparation of $\left[\mathrm{M}(2-\mathrm{ab})_{2}\left(\mathrm{~N}_{2} \mathrm{H}_{4}\right)\right] \cdot 3 \mathrm{H}_{2} \mathrm{O}$ was achieved by adding a ligand solution prepared by mixing $\mathrm{N}_{2} \mathrm{H}_{4}(0.2 \mathrm{~mL}, 4 \mathrm{mmol})$ with an aqueous solution of $2-\mathrm{abH}(0.180 \mathrm{~g}, 1 \mathrm{mmol}$ in 60 $\mathrm{mL}$ of $\mathrm{H}_{2} \mathrm{O}$ ) to a freshly prepared metal nitrate solution (for example, $\mathrm{Ni}\left(\mathrm{NO}_{3}\right)_{2} \cdot 6 \mathrm{H}_{2} \mathrm{O}, 0.291 \mathrm{~g}, 1$ mmol in $20 \mathrm{~mL}$ of $\mathrm{H}_{2} \mathrm{O}$ ) followed by stirring the reaction mixture vigorously and the $\mathrm{pH}$ was found to be 6 . Then the reaction mixture was kept over hot water bath at $80{ }^{\circ} \mathrm{C}$. A microcrystalline product ( $\mathrm{Ni}$ : pale blue, $\mathrm{Co}$ : pink, $\mathrm{Cd}$ and $\mathrm{Zn}$ colourless) was separated out from the mixture within 30 min which was filtered, washed with distilled water and ether and then dried in air.

All other complexes of $2-\mathrm{abH}$ and $3-\mathrm{abH}$ were obtained by adopting similar procedure as described above. 
Preparation of $\left[\mathrm{M}(4-\mathrm{ab})_{2}\left(\mathrm{~N}_{2} \mathrm{H}_{4}\right)_{2}\right] \cdot 2 \mathrm{H}_{2} \mathrm{O}$ where $\mathrm{M}=\mathrm{Co}, \mathrm{Ni}, \mathrm{Zn}$ and $\mathrm{Cd}$

Hydrazine transition metal complexes of $4-\mathrm{abH}$ were prepared by adding the ligand solution to corresponding hydrated metal nitrate solution with constant stirring at $\mathrm{pH} 5$. Among them $\mathrm{Ni}$, $\mathrm{Cd}$ and $\mathrm{Zn}$ complexes were formed in the molar ratio, metal : acid : base $=1: 1: 2$ while Co at 1:2:6. Nickel and cobalt complexes were obtained in water medium, whereas cadmium and zinc complexes were recovered using 1:1 aqueous ethanol.

Preparation of $\left[\mathrm{Cd}\left(\mathrm{H}_{2} \mathrm{O}\right)_{3}\left(\left(\mathrm{C}_{6} \mathrm{H}_{4}(4-\mathrm{OH})(\mathrm{COO})\right)_{2}\right] \cdot \mathrm{H}_{2} \mathrm{O}\right.$

The title compound was prepared by the reaction of 4-acetoxy benzoic acid, hydrazine hydrate and cadmium nitrate tetra hydrate in aqueous medium. Initially the acid (1.8 g, $10 \mathrm{mmol})$ was mixed in $40 \mathrm{~mL}$ of water and hydrazine hydrate $(1 \mathrm{~g}, 20 \mathrm{mmol})$ was added to dissolve the acid. A complete dissolution occurred on heating the solution at $80{ }^{\circ} \mathrm{C}$ over a hot water bath for 10 min. To this hot solution, an aqueous solution of the cadmium nitrate tetrahydrate $(3.08 \mathrm{~g}, 10$ mmol in $20 \mathrm{~mL}$ of water) was added at $\mathrm{pH} 5$ and heated again at the same temperature for about $6 \mathrm{~h}$ till a thick viscous solution was formed. On adding absolute ethanol $(50 \mathrm{~mL})$, a white precipitate $\left(\left[\mathrm{Cd}(4-\mathrm{ab})_{2}\left(\mathrm{~N}_{2} \mathrm{H}_{4}\right)_{2}\right] \cdot 2 \mathrm{H}_{2} \mathrm{O}\right)$ formed immediately was filtered and dried. On slow evaporation of the filtrate, colorless prismatic crystals of the title compound were obtained in 15 days, which was characterized by single crystal XRD-studies.

\section{Physico-chemical methods}

The hydrazine content in all the complexes was determined volumetrically using $0.025 \mathrm{M}$ potassium iodate solution under Andrews' conditions [31]. The metal content was determined by EDTA complexometric titration [31] after decomposing a known weight of the sample with $1: 1 \mathrm{HNO}_{3}$. Magnetic measurements were carried out by the Guoy method using $\mathrm{Hg}\left[\mathrm{Co}(\mathrm{NCS})_{4}\right]$ as calibrant. The electronic spectra for solid-state complexes were obtained using a Varian, Cary 5000 recording spectrophotometer.

Infra-red spectra were recorded using KBR disc $\left(4000-400 \mathrm{~cm}^{-1}\right)$ on a Shimadzu FTIR-8201 (PC) S spectrophotometer. The simultaneous TG-DTA studies were done on a Perkin Elmer, Diamond TG/DTA analyzer and the curves were obtained in static air using 5-10 mg of the samples at the heating rate of $10{ }^{\circ} \mathrm{C} / \mathrm{min}$. The XRD patterns were recorded on a Bruker AXS D8 Advance diffractometer with an X-ray source $\mathrm{Cu}$, wavelength $1.5406 \AA$ using a $\mathrm{Si}(\mathrm{Li}) \mathrm{PSD}$ detector. The elemental analysis was carried out using a CHNS Elementar Vario EL III Elemental Analyzer.

\section{RESULTS AND DISCUSSION}

All the complexes obtained were polycrystalline powders, which are stable in air and insensitive to light. They are insoluble in ethanol, ether and benzene. The analytical data of the complexes given in Table 1 were compatible with the proposed compositions for the complexes.

\section{Electronic spectra and magnetic susceptibility measurements}

The compounds were insoluble in both water and organic solvents. Hence their electronic reflectance spectra were recorded for solid samples. The absorptions 22422-17857, 1644711933, 8779-5482 $\mathrm{cm}^{-1}$ are assigned to the transitions: ${ }^{4} \mathrm{~T}_{1 \mathrm{~g}}(\mathrm{~F}) \rightarrow{ }^{4} \mathrm{~T}_{1 \mathrm{~g}}(\mathrm{P}),{ }^{4} \mathrm{~T}_{1 \mathrm{~g}} \rightarrow{ }^{4} \mathrm{~T}_{1 \mathrm{~g}}(\mathrm{P}),{ }^{4} \mathrm{~T}_{1 \mathrm{~g}} \rightarrow$ ${ }^{4} \mathrm{~A}_{2 \mathrm{~g}}$, and ${ }^{4} \mathrm{~A}_{2 \mathrm{~g}} \rightarrow{ }^{4} \mathrm{~T}_{2 \mathrm{~g}}$ and the values $21142,20000-13587,8467-7128 \mathrm{~cm}^{-1}$ are assigned to ${ }^{3} \mathrm{~A}_{2 \mathrm{~g}}$ $\rightarrow{ }^{3} \mathrm{~T}_{1 \mathrm{~g}},{ }^{3} \mathrm{~T}_{1 \mathrm{~g}}(\mathrm{P}) \rightarrow{ }^{3} \mathrm{~T}_{2 \mathrm{~g}}$ and ${ }^{3} \mathrm{~A}_{2 \mathrm{~g}}(\mathrm{~F}) \rightarrow{ }^{3} \mathrm{~T}_{1 \mathrm{~g}}(\mathrm{P})$ for cobalt and nickel complexes, respectively. These assignments evidence the distorted octahedral geometry of the complexes [28, 29, 32]. 
The effective magnetic moments of the cobalt and nickel complexes lie in the range of 4.7-5.2 $\mathrm{BM}$ and 2.8-3.3 BM, respectively. The values of cobalt(II) complexes indicated that $\mathrm{Co}^{2+}$ is in high spin state. The electronic spectrum of $\left[\mathrm{Ni}(2-\mathrm{ab})_{2}\left(\mathrm{~N}_{2} \mathrm{H}_{4}\right)\right] \cdot 3 \mathrm{H}_{2} \mathrm{O}$ is given as representative image in Figure 1.

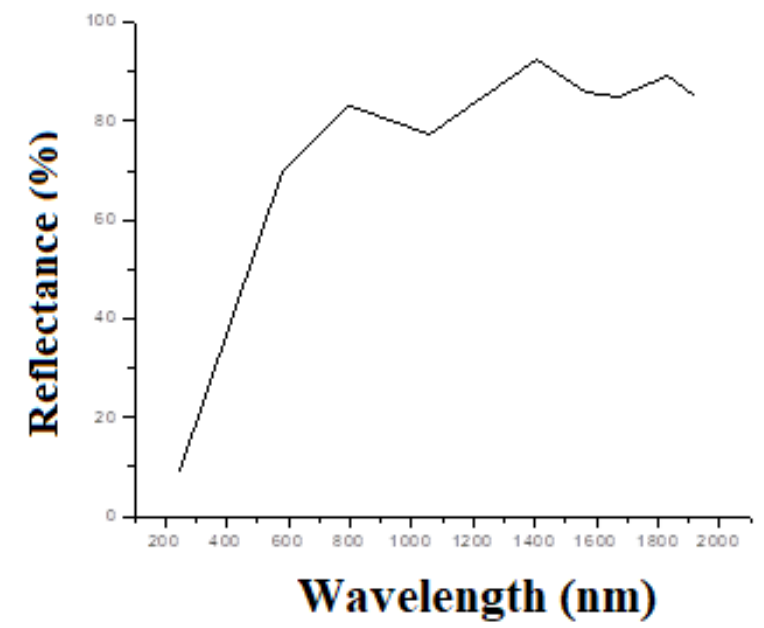

Figure 1. Electronic spectrum of $\left[\mathrm{Ni}\left\{2-\mathrm{C}_{6} \mathrm{H}_{4}\left(\mathrm{OCOCH}_{3}\right) \mathrm{COO}\right\}_{2}\left(\mathrm{~N}_{2} \mathrm{H}_{4}\right) \cdot 2 \mathrm{H}_{2} \mathrm{O}\right] \cdot \mathrm{H}_{2} \mathrm{O}$.

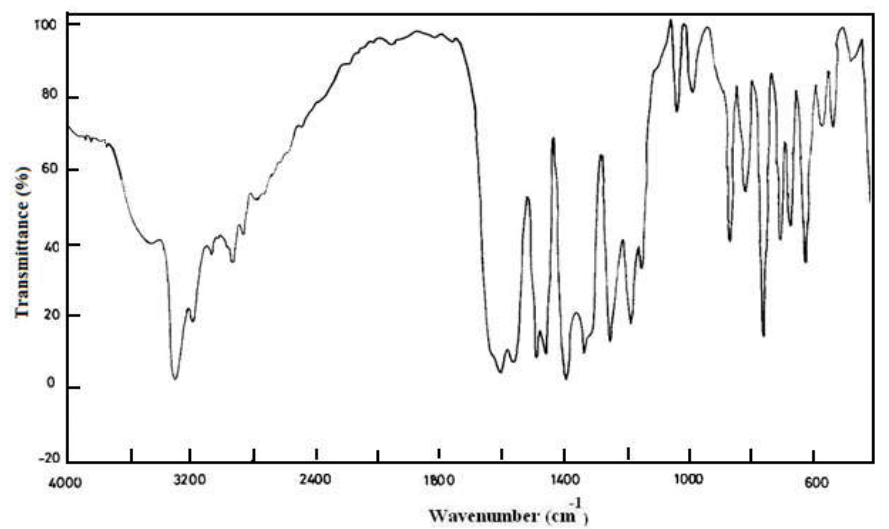

Figure 2. IR spectrum of $\left[\mathrm{Ni}\left\{2-\mathrm{C}_{6} \mathrm{H}_{4}\left(\mathrm{OCOCH}_{3}\right) \mathrm{COO}\right\}_{2}\left(\mathrm{~N}_{2} \mathrm{H}_{4}\right) \cdot 2 \mathrm{H}_{2} \mathrm{O}\right] \cdot \mathrm{H}_{2} \mathrm{O}$.

IR spectra of complexes

IR spectroscopic values of all the complexes are listed in Table 1. The IR spectra of complexes displayed peaks in the range of 1599-1682 and 1391-1454 $\mathrm{cm}^{-1}$ corresponding to the asymmetric and symmetric $\mathrm{C}=\mathrm{O}$ stretching frequencies of the metal bound carboxylates. The difference between $v_{\mathrm{C}=\text { Oasym }}$ and $v_{\mathrm{C}=\mathrm{O} \text { sym }}$ values was found to be in the range of $172-228 \mathrm{~cm}^{-1}$, which indicated the monodentate coordination of carboxyl group with metal. The complexes displayed the N-N stretching frequency of hydrazine in the range $966-988 \mathrm{~cm}^{-1}$ which implied that hydrazine was involved in bridged bidentate coordination with the metal ion. The O-H stretch of

Bull. Chem. Soc. Ethiop. 2021, 35(2) 
water molecules were noticed between 3329 and $3471 \mathrm{~cm}^{-1}$ in all complexes. An additional band observed in the range $818-862 \mathrm{~cm}^{-1}$ also supported the presence of lattice water molecules [33]. $v_{\mathrm{N}-\mathrm{H}}$ bands were found to merged with $\mathrm{O}-\mathrm{H}$ stretching and seen in the region of $3250-3331 \mathrm{~cm}^{-1}$. The IR spectrum also displayed bands in the range $1705-1777 \mathrm{~cm}^{-1}$ for $v_{\mathrm{C}=\mathrm{O}}$ of acetoxy group, indicating that the acetoxy group was not involved in complexation, due to the fact that the acetoxy group $\left(-\mathrm{O}_{\mathrm{COCH}}\right)$, is a weak electron contributor [34]. The IR spectrum of the complex $\left[\mathrm{Ni}\left\{2-\mathrm{C}_{6} \mathrm{H}_{4}\left(\mathrm{OCOCH}_{3}\right) \mathrm{COO}\right\}_{2}\left(\mathrm{~N}_{2} \mathrm{H}_{4}\right) \cdot 2 \mathrm{H}_{2} \mathrm{O}\right] \cdot \mathrm{H}_{2} \mathrm{Ois}$ shown in Figure 2.

Table 1 Analytical and IR data transition metal complexes of isomeric acetoxy benzoic acids and hydrazine.

\begin{tabular}{|c|c|c|c|c|c|c|c|c|c|c|c|c|}
\hline \multirow[b]{2}{*}{ Compound } & \multicolumn{5}{|c|}{ Analytical data (\%) } & \multicolumn{7}{|c|}{ IR data $\left(\mathrm{cm}^{-1}\right) \mathrm{b}=$ broad; $\mathrm{s}=$ sharp; $\mathrm{m}=$ medium } \\
\hline & \begin{tabular}{|c|} 
Carbon \\
found \\
(cald.)
\end{tabular} & $\begin{array}{l}\text { Hydrogen } \\
\text { found } \\
\text { (cald.) }\end{array}$ & $\begin{array}{c}\text { Nitrogen } \\
\text { found } \\
\text { (cald.) }\end{array}$ & $\begin{array}{c}\text { Hydrazine } \\
\text { found } \\
\text { (cald.) }\end{array}$ & $\begin{array}{l}\text { Metal } \\
\text { found } \\
\text { (cald.) }\end{array}$ & $v_{\mathrm{N}-\mathrm{N}}$ & $v_{\mathrm{OH}}$ & $\begin{array}{c}v_{\mathrm{C}=\mathrm{O}} \\
\mathrm{sym}\end{array}$ & $\begin{array}{c}v_{\mathrm{C}=\mathrm{O}} \\
\text { asym }\end{array}$ & $v \mathrm{H}_{2} \mathrm{O}$ & $v_{\mathrm{N}-\mathrm{H}}$ & $\begin{array}{c}v_{\mathrm{C}=\mathrm{O}} \\
\text { (acetoxy } \\
\text { group ) }\end{array}$ \\
\hline \multicolumn{13}{|c|}{ 2-abH complexes } \\
\hline $\begin{array}{l}{\left[\mathrm{Ni}\left\{2-{ }_{6} \mathrm{H}_{4}\left(\mathrm{OCOCH}_{3}\right)\right.\right.} \\
\left.\mathrm{COO}\}_{2}\left(\mathrm{~N}_{2} \mathrm{H}_{4}\right) 2 \mathrm{H}_{2} \mathrm{O}\right] \cdot \mathrm{H}_{2} \mathrm{O}\end{array}$ & $\begin{array}{c}42.93 \\
(42.94)\end{array}$ & $\begin{array}{c}4.80 \\
(4.81)\end{array}$ & $\begin{array}{c}5.58 \\
(5.57)\end{array}$ & $\begin{array}{c}6.37 \\
(6.36)\end{array}$ & $\begin{array}{c}11.69 \\
(11.67)\end{array}$ & $980 \mathrm{~s}$ & $3449 \mathrm{~m}$ & $1410 \mathrm{~s}$ & $1605 \mathrm{~s}$ & $567 \mathrm{~s}$ & $3295 \mathrm{~s}$ & $1757 \mathrm{~m}$ \\
\hline $\begin{array}{l}{\left[\mathrm{Co}\left\{2-\mathrm{C}_{6} \mathrm{H}_{4}\left(\mathrm{OCOCH}_{3}\right)\right.\right.} \\
\left.\mathrm{COO}\}_{2}\left(\mathrm{~N}_{2} \mathrm{H}_{4}\right) 2 \mathrm{H}_{2} \mathrm{O}\right] \cdot \mathrm{H}_{2} \mathrm{O}\end{array}$ & $\begin{array}{c}42.91 \\
(42.92)\end{array}$ & $\begin{array}{c}4.80 \\
(4.80)\end{array}$ & $\begin{array}{c}5.57 \\
(5.56)\end{array}$ & $\begin{array}{c}6.36 \\
(6.36)\end{array}$ & $\begin{array}{c}11.70 \\
(11.71)\end{array}$ & 970s & $3420 \mathrm{~m}$ & $1391 \mathrm{~s}$ & $1605 \mathrm{~s}$ & $590 \mathrm{~s}$ & $298 \mathrm{~s}$ & $1760 \mathrm{~b}$ \\
\hline $\begin{array}{l}\overline{[C d}\left\{2-\mathrm{C}_{6} \mathrm{H}_{4}\left(\mathrm{OCOCH}_{3}\right)\right. \\
\left.\mathrm{COO}\}_{2}\left(\mathrm{~N}_{2} \mathrm{H}_{4}\right) 2 \mathrm{H}_{2} \mathrm{O}\right] \cdot \mathrm{H}_{2} \mathrm{O}\end{array}$ & $\begin{array}{c}38.80 \\
(38.80)\end{array}$ & $\begin{array}{c}4.34 \\
(4.35)\end{array}$ & $\begin{array}{c}5.02 \\
(5.03)\end{array}$ & $\begin{array}{c}5.74 \\
(5.75)\end{array}$ & $\begin{array}{c}20.18 \\
(20.19)\end{array}$ & $966 \mathrm{~s}$ & $3448 b$ & $1454 \mathrm{~s}$ & $1626 \mathrm{~s}$ & $563 \mathrm{~s}$ & $3315 \mathrm{~s}$ & $1763 b$ \\
\hline $\begin{array}{l}{\left[\mathrm{Zn}\left\{2-\mathrm{C}_{6} \mathrm{H}_{4}(\mathrm{C}\right.\right.} \\
\left.\mathrm{COO}\}_{2} \mathrm{~N}_{2} \mathrm{H}_{4}\right)\end{array}$ & $\begin{array}{c}42.39 \\
(42.37)\end{array}$ & $\begin{array}{c}4.73 \\
(4.74)\end{array}$ & $\begin{array}{c}5.49 \\
(5.49)\end{array}$ & $\begin{array}{c}6.26 \\
(6.28)\end{array}$ & $\begin{array}{c}12.84 \\
(12.83) \\
\end{array}$ & $964 s$ & $3443 b$ & $1406 \mathrm{~m}$ & $1605 \mathrm{~s}$ & $571 \mathrm{~s}$ & $3262 \mathrm{~m}$ & $1763 \mathrm{~s}$ \\
\hline \multicolumn{13}{|c|}{ 3-abH complexes } \\
\hline $\begin{array}{l}{\left[\mathrm{Ni}\left\{3-6 \mathrm{H}_{4}\left(\mathrm{OCOCH}_{3}\right)\right.\right.} \\
\left.\mathrm{COO}\}_{2}\left(\mathrm{~N}_{2} \mathrm{H}_{4}\right) 2 \mathrm{H}_{2} \mathrm{O}\right] \cdot \mathrm{H}_{2} \mathrm{O}\end{array}$ & $\begin{array}{c}42.92 \\
(42.94)\end{array}$ & $\begin{array}{c}4.79 \\
(4.81)\end{array}$ & $\begin{array}{c}5.57 \\
(5.57)\end{array}$ & $\begin{array}{c}6.36 \\
(6.36)\end{array}$ & $\mid \begin{array}{c}11.65 \\
(11.67)\end{array}$ & $988 \mathrm{~m}$ & $3356 \mathrm{~m}$ & $1447 \mathrm{~s}$ & $1620 \mathrm{~m}$ & $567 \mathrm{~m}$ & $3298 \mathrm{~s}$ & $1705 \mathrm{~m}$ \\
\hline $\begin{array}{l}{\left[\mathrm{Co}\left\{3-\mathrm{C}_{6} \mathrm{H}_{4}\left(\mathrm{OCOCH}_{3}\right)\right.\right.} \\
\left.\mathrm{COO}\}_{2}\left(\mathrm{~N}_{2} \mathrm{H}_{4}\right) 2 \mathrm{H}_{2} \mathrm{O}\right] . \mathrm{H}_{2} \mathrm{O}\end{array}$ & $\begin{array}{c}42.93 \\
(42.92)\end{array}$ & $\begin{array}{c}4.81 \\
(4.80)\end{array}$ & $\begin{array}{c}5.58 \\
(5.56)\end{array}$ & $\begin{array}{c}6.37 \\
(6.36)\end{array}$ & $\begin{array}{c}11.74 \\
(11.71)\end{array}$ & $980 \mathrm{~m}$ & $3356 \mathrm{~b}$ & $1404 s$ & $1614 s$ & $611 \mathrm{~s}$ & $3250 \mathrm{~s}$ & $1705 \mathrm{~m}$ \\
\hline $\begin{array}{l}\mathrm{Cd}\left\{3{ }_{6} \mathrm{H}_{4}\left(\mathrm{OCOCH}_{3}\right)\right. \\
\left.\mathrm{COO}\}_{2}\left(\mathrm{~N}_{2} \mathrm{H}_{4}\right) 2 \mathrm{H}_{2} \mathrm{O}\right] \cdot \mathrm{H}_{2} \mathrm{O}\end{array}$ & $\begin{array}{c}38.79 \\
(38.80)\end{array}$ & $\begin{array}{c}4.36 \\
(4.35)\end{array}$ & $\begin{array}{c}5.01 \\
(5.03)\end{array}$ & $\begin{array}{c}5.76 \\
(5.75)\end{array}$ & $\begin{array}{c}20.20 \\
(20.19) \\
\end{array}$ & $980 \mathrm{~m}$ & $3329 \mathrm{~m}$ & $1454 \mathrm{~s}$ & $1682 \mathrm{~m}$ & $623 \mathrm{~s}$ & $3271 \mathrm{~m}$ & $1777 \mathrm{~m}$ \\
\hline $\begin{array}{l}{\left[\mathrm{Zn}\left\{3-6 \mathrm{H}_{4}\left(\mathrm{OCOCH}_{3}\right)\right.\right.} \\
\left.\mathrm{COO}\}_{2}\left(\mathrm{~N}_{2} \mathrm{H}_{4}\right) 2 \mathrm{H}_{2} \mathrm{O}\right] \cdot \mathrm{H}_{2} \mathrm{O}\end{array}$ & $\begin{array}{c}42.38 \\
(42.37) \\
\end{array}$ & $\begin{array}{c}4.72 \\
(4.74)\end{array}$ & $\begin{array}{c}5.48 \\
(5.49)\end{array}$ & $\begin{array}{c}6.27 \\
(6.28)\end{array}$ & $\begin{array}{c}12.84 \\
(12.83)\end{array}$ & $974 s$ & $3456 \mathrm{~b}$ & $1425 b$ & $1609 \mathrm{~s}$ & $621 \mathrm{~s}$ & $3331 b$ & $1775 \mathrm{~s}$ \\
\hline \multicolumn{13}{|c|}{ 4-abH complexes } \\
\hline $\begin{array}{l}{\left[\mathrm{Ni}\left\{4-{ }_{6} \mathrm{H}_{4}\left(\mathrm{OCOCH}_{3}\right)\right.\right.} \\
\left.\mathrm{COO}_{\}_{2}}\left(\mathrm{~N}_{2} \mathrm{H}_{4}\right)\right] \cdot 2 \mathrm{H}_{2} \mathrm{O}\end{array}$ & $\begin{array}{c}41.80 \\
(41.78)\end{array}$ & $\begin{array}{c}5.05 \\
(5.07)\end{array}$ & $\begin{array}{c}10.81 \\
(10.83)\end{array}$ & $\begin{array}{c}12.40 \\
(12.38)\end{array}$ & $\begin{array}{c}11.33 \\
(11.35)\end{array}$ & $983 \mathrm{~s}$ & $3471 b$ & $1443 \mathrm{~s}$ & $1626 b$ & $624 s$ & $3320 \mathrm{~b}$ & $1750 \mathrm{~b}$ \\
\hline $\begin{array}{l}{\left[\mathrm{Co}\left\{4-{ }_{6} \mathrm{H}_{4}\left(\mathrm{OCOCH}_{3}\right)\right.\right.} \\
\left.\mathrm{COO}\}_{2}\left(\mathrm{~N}_{2} \mathrm{H}_{4}\right)\right] \cdot 2 \mathrm{H}_{2} \mathrm{O}\end{array}$ & $\begin{array}{c}41.76 \\
(41.76)\end{array}$ & $\begin{array}{c}5.08 \\
(5.07)\end{array}$ & $\begin{array}{c}10.82 \\
(10.83) \\
\end{array}$ & $\begin{array}{c}12.70 \\
(12.37)\end{array}$ & $\begin{array}{c}11.44 \\
(11.39)\end{array}$ & $973 \mathrm{~s}$ & $3437 b$ & $1405 \mathrm{~s}$ & $1599 b$ & $625 \mathrm{~s}$ & $3323 b$ & $1768 \mathrm{~s}$ \\
\hline $\begin{array}{l}{\left[\mathrm{Cd}\left\{4-{ }_{6} \mathrm{H}_{4}\left(\mathrm{OCOCH}_{3}\right)\right.\right.} \\
\left.\mathrm{COO}_{2}\left(\mathrm{~N}_{2} \mathrm{H}_{4}\right)\right] \cdot 2 \mathrm{H}_{2} \mathrm{O}\end{array}$ & $\begin{array}{c}37.83 \\
(37.84) \\
\end{array}$ & $\begin{array}{c}4.55 \\
(4.59) \\
\end{array}$ & $\begin{array}{c}9.82 \\
(9.81) \\
\end{array}$ & $\begin{array}{c}11.20 \\
(11.21) \\
\end{array}$ & $\begin{array}{c}20.40 \\
(19.70) \\
\end{array}$ & $971 \mathrm{~s}$ & $3469 b$ & $1426 b$ & $1622 b$ & $616 \mathrm{~s}$ & $3324 b$ & $1771 \mathrm{~s}$ \\
\hline $\begin{array}{l}\mathrm{Zn}\left\{4-\mathrm{C}_{6} \mathrm{H}_{4}\left(\mathrm{OCOCH}_{3}\right)\right. \\
\left.\mathrm{COO}\}_{2}\left(\mathrm{~N}_{2} \mathrm{H}_{4}\right)_{2}\right] \cdot 2 \mathrm{H}_{2} \mathrm{O}\end{array}$ & $\begin{array}{c}43.91 \\
(43.92)\end{array}$ & $\begin{array}{c}5.12 \\
(5.13)\end{array}$ & $\begin{array}{c}5.60 \\
(5.70)\end{array}$ & $\begin{array}{c}6.47 \\
(6.50)\end{array}$ & \begin{tabular}{|c|}
13.29 \\
$(13.30)$
\end{tabular} & $978 \mathrm{~m}$ & $3454 b$ & $1447 \mathrm{~b}$ & $1626 \mathrm{~b}$ & $574 b$ & $3321 b$ & $1770 \mathrm{~s}$ \\
\hline
\end{tabular}

In the IR spectrum of the compound, the bidendate coordination of carboxylate group was understood by observing bands of $v_{\mathrm{C}=\text { Oasym }}$ at $1514 \mathrm{~cm}^{-1}$ and $v_{\mathrm{C}=\mathrm{Osym}}$ at $1389 \mathrm{~cm}^{-1}$, respectively, and the difference being $125 \mathrm{~cm}^{-1}$ which supports the bidentate coordination of the carboxylate group with metal ion. The presence of lattice water was revealed by the modes $3447,1672,1599$ and $1514 \mathrm{~cm}^{-1}$. The coordinated water molecules were found by the bands at 844,775 and 617 $\mathrm{cm}^{-1}$ and these values are similar to that reported in the literature [33]. The bands at $499 \mathrm{~cm}^{-1}$ and $543 \mathrm{~cm}^{-1}$ showed that the oxygen was coordinated with metal, which were also similar to that reported [33].

Thermal analysis of complexes

The TG-DTA of compounds were performed in the temperature range $30-700{ }^{\circ} \mathrm{C}$ in static air atmosphere and the thermal degradation data is given in Table 2. The TG-DTA traces of $\left(\left[\mathrm{Ni}\left\{(2-\mathrm{ab})_{2}\left(\mathrm{~N}_{2} \mathrm{H}_{4}\right)\right\}\right] \cdot 3 \mathrm{H}_{2} \mathrm{O},\left[\mathrm{Ni}\left\{(3-\mathrm{ab})_{2}\left(\mathrm{~N}_{2} \mathrm{H}_{4}\right)\right\}\right] \cdot 3 \mathrm{H}_{2} \mathrm{O}\right.$ and $\left[\mathrm{Ni}(4-\mathrm{ab})_{2}\left(\mathrm{~N}_{2} \mathrm{H}_{4}\right)_{2}\right] \cdot 2 \mathrm{H}_{2} \mathrm{O}$, are given as representative examples in Figure 3. 


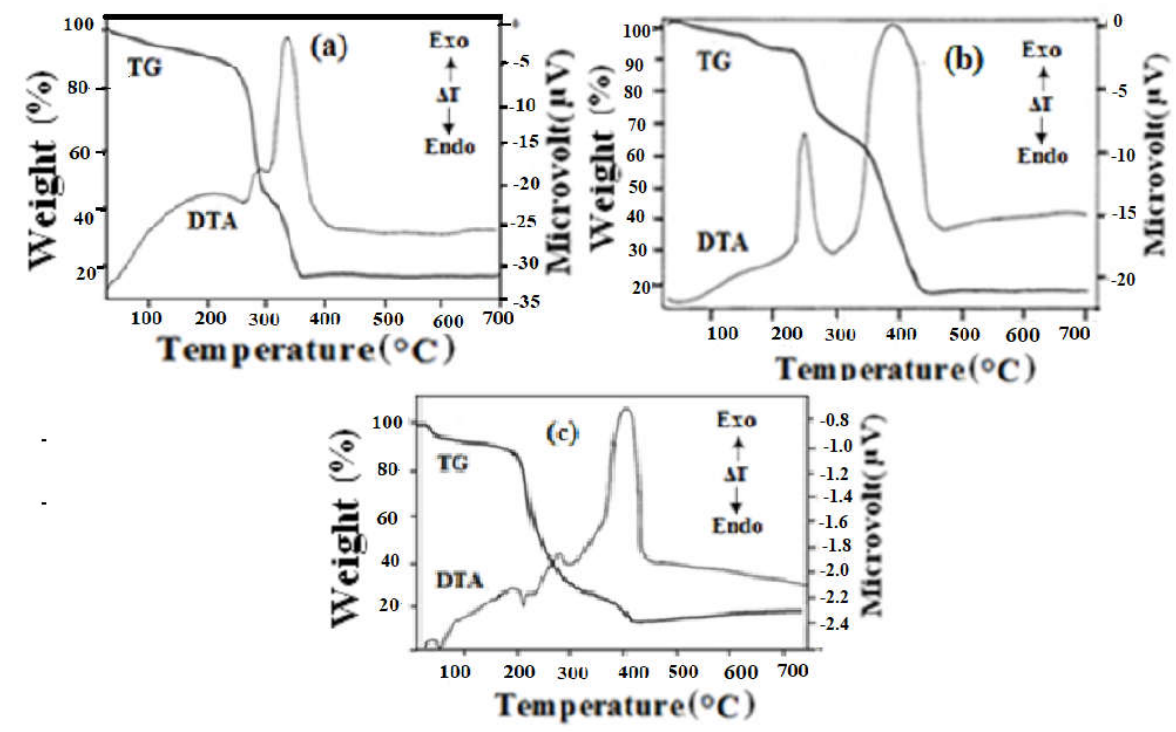

Figure 3. TG-DTA curves of:

(a) $\left[\mathrm{Ni}\left\{2-\mathrm{C}_{6} \mathrm{H}_{4}\left(\mathrm{OCOCH}_{3}\right) \mathrm{COO}\right\}_{2}\left(\mathrm{~N}_{2} \mathrm{H}_{4}\right) \cdot 2 \mathrm{H}_{2} \mathrm{O}\right] \cdot \mathrm{H}_{2} \mathrm{O}$,

(b) $\left[\mathrm{Ni}\left\{3-\mathrm{C}_{6} \mathrm{H}_{4}\left(\mathrm{OCOCH}_{3}\right) \mathrm{COO}\right\}_{2}\left(\mathrm{~N}_{2} \mathrm{H}_{4}\right) \cdot 2 \mathrm{H}_{2} \mathrm{O}\right] \cdot \mathrm{H}_{2} \mathrm{O}$ and

(c) $\left[\mathrm{Ni}\left\{\underline{4}-\mathrm{C}_{6} \mathrm{H}_{4}\left(\mathrm{OCOCH}_{3}\right) \mathrm{COO}\right\}_{2}\left(\mathrm{~N}_{2} \mathrm{H}_{4}\right)_{2}\right] \cdot 2 \mathrm{H}_{2} \mathrm{O}$.

Thermal data of 2-abH and 3-abH complexes

$\mathrm{Co}, \mathrm{Ni}, \mathrm{Zn}$ and $\mathrm{Cd}$ complexes of 2-abH and 4-abH and $\mathrm{Co}$, Ni and $\mathrm{Cd}$ complexes of 3-abH showed three-step decomposition. They showed endothermic dehydration in the range 80-100 ${ }^{\circ} \mathrm{C}$ and exothermic dehydrazination in the temperature range of $210-280{ }^{\circ} \mathrm{C}$. Further, these metal carboxylates were found to undergo oxidative decomposition to form their respective metal oxide residue, displaying the exotherms between 350 and $490{ }^{\circ} \mathrm{C}$. Our effort to isolate the intermediate from these complexes was unsuccessful, due to their continuous decomposition as evident from their TG.

The dehydrazinated cobalt carboxylate 4-abH underwent exothermic decomposition to form metal phthalate intermediate at $350{ }^{\circ} \mathrm{C}$, and this intermediate further continued to decompose to form its metal oxide residue in the temperature range of $400-467{ }^{\circ} \mathrm{C}$. This decomposition was found to match with the literature reported [35].

$\mathrm{Zn}$ complex of $3-\mathrm{abH}$ showed two exotherms at 280 and $440{ }^{\circ} \mathrm{C}$ indicating its oxidative decomposition to form its metal oxide, whereas the $\mathrm{Zn}$ complex of 4-abH directly decomposed to metal oxide at $271{ }^{\circ} \mathrm{C}$ exothermally indicating its high instability behavior. In both of these zinc complexes neither dehydration nor dehydrazination stages were clear. The thermal degradation patterns are given by the following reactions:

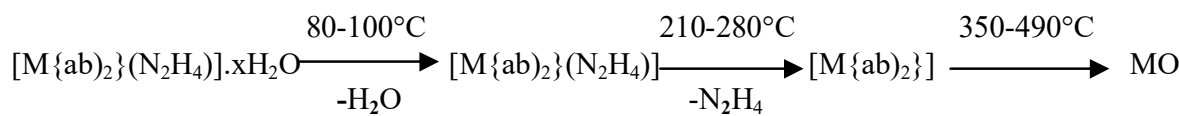

(where $\mathrm{x}=3 ; \mathrm{M}=\mathrm{Co}, \mathrm{Ni}, \mathrm{Cd}$ and $\mathrm{Zn}$ of $2-\mathrm{abH}$ complexes, $\mathrm{x}=3,2$ and $\mathrm{M}=\mathrm{Co}$, Ni and $\mathrm{Cd}$ of 3-abH and 4-abH complexes).

Bull. Chem. Soc. Ethiop. 2021, 35(2) 


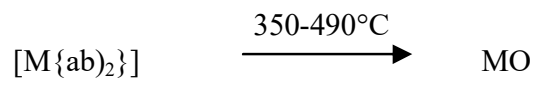

(where $\mathrm{M}=\mathrm{Co}, \mathrm{Ni}, \mathrm{Cd}$ and $\mathrm{Zn}$ of 2-abH; $\mathrm{M}=\mathrm{Co}$, Ni and $\mathrm{Cd}$ of $3-\mathrm{abH}$ and $\mathrm{M}=\mathrm{Ni}$ and $\mathrm{Cd}$ of 4-abH).

$\left.\left[\mathrm{Co}\{\mathrm{ab})_{2}\right\}\right]\left[\mathrm{Co}\left\{\mathrm{C}_{6} \mathrm{H}_{4}(\mathrm{COO})_{2}\right\}\right] \stackrel{350^{\circ} \mathrm{C}}{\longrightarrow} \mathrm{CoO}$ (complex of4-abH)

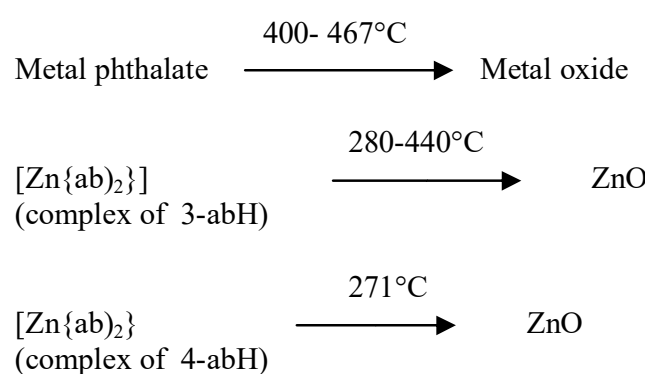

Table 2. Thermal data of transition metal complexes of isomeric acetoxy benzoic acids and hydrazine.

\begin{tabular}{|c|c|c|c|c|c|}
\hline \multirow{3}{*}{ Complex } & \multirow{3}{*}{$\begin{array}{l}\text { DTA Temp } \\
\left({ }^{\circ} \mathrm{C}\right)\end{array}$} & \multicolumn{3}{|c|}{ Thermogravimetry } & \multirow{3}{*}{ Nature of the reaction } \\
\hline & & \multirow[t]{2}{*}{$\begin{array}{c}\text { Temp. } \\
\text { range, } \\
\left({ }^{\circ} \mathrm{C}\right)\end{array}$} & \multicolumn{2}{|c|}{ Weight loss $\%$} & \\
\hline & & & Obsd. & Cald. & \\
\hline \multirow{4}{*}{$\begin{array}{l}{\left[\mathrm{Ni}\left\{2-\mathrm{C}_{6} \mathrm{H}_{4}\left(\mathrm{OCOCH}_{3}\right)\right.\right.} \\
\left.\mathrm{COO}\}_{2}\left(\mathrm{~N}_{2} \mathrm{H}_{4}\right) \cdot 2 \mathrm{H}_{2} \mathrm{O}\right] \cdot \mathrm{H}_{2} \mathrm{O}\end{array}$} & $90(+)$ & $80-150$ & 10.5 & 10.7 & Dehydration \\
\hline & $210(-)]$ & $150-240$ & 17.3 & 17.1 & Dehydrazination \\
\hline & $300(-)$ & & & & \\
\hline & $350(-)$ & $240-600$ & 85.0 & 85.2 & Decomposition to metal oxide \\
\hline \multirow{3}{*}{$\begin{array}{l}{\left[\mathrm{Co}\left\{2-\mathrm{C}_{6} \mathrm{H}_{4}\left(\mathrm{OCOCH}_{3}\right)\right.\right.} \\
\left.\mathrm{COO}\}_{2}\left(\mathrm{~N}_{2} \mathrm{H}_{4}\right) \cdot 2 \mathrm{H}_{2} \mathrm{O}\right] \cdot \mathrm{H}_{2} \mathrm{O}\end{array}$} & $\left.\begin{array}{r}100(+) \\
190(+)\end{array}\right\}$ & $40-200$ & 10.6 & 10.7 & Dehydration \\
\hline & & $200-280$ & & & Dehydrazination \\
\hline & $\begin{array}{l}230(-) \\
360(-)\end{array}$ & $280-600$ & $\begin{array}{l}17.0 \\
85.0\end{array}$ & $\begin{array}{l}17.1 \\
85.1\end{array}$ & Decomposition to metal oxide \\
\hline \multirow{4}{*}{$\begin{array}{l}{\left[\mathrm{Cd}\left\{2-\mathrm{C}_{6} \mathrm{H}_{4}\left(\mathrm{OCOCH}_{3}\right)\right.\right.} \\
\left.\mathrm{COO}\}_{2}\left(\mathrm{~N}_{2} \mathrm{H}_{4}\right) \cdot 2 \mathrm{H}_{2} \mathrm{O}\right] \cdot \mathrm{H}_{2} \mathrm{O}\end{array}$} & $95(+)$ & & & & \\
\hline & $190(+)$ & 80-198 & 10.0 & 9.7 & Dehydration \\
\hline & $210(-)$ & $198-240$ & 15.2 & 15.4 & Dehydrazination \\
\hline & $480(-)$ & $240-600$ & 77.0 & 76.9 & Decomposition to metal oxide \\
\hline \multirow{4}{*}{$\begin{array}{l}{\left[\mathrm{Zn}\left\{2-\mathrm{C}_{6} \mathrm{H}_{4}\left(\mathrm{OCOCH}_{3}\right)\right.\right.} \\
\left.\mathrm{COO}\}_{2}\left(\mathrm{~N}_{2} \mathrm{H}_{4}\right) \cdot 2 \mathrm{H}_{2} \mathrm{O}\right] \cdot \mathrm{H}_{2} \mathrm{O}\end{array}$} & $90(+) 7$ & & & & \\
\hline & $200(+)$ & $75-210$ & 10.5 & 10.6 & Dehydration \\
\hline & $278(-)$ & $210-260$ & 17.0 & 16.9 & Dehydrazination \\
\hline & $420(-)$ & $260-600$ & 83.9 & 84.0 & Decomposition to metal oxide \\
\hline \multirow{3}{*}{$\begin{array}{l}{\left[\mathrm{Ni}\left\{3-\mathrm{C}_{6} \mathrm{H}_{4}\left(\mathrm{OCOCH}_{3}\right)\right.\right.} \\
\left.\mathrm{COO}\}_{2}\left(\mathrm{~N}_{2} \mathrm{H}_{4}\right) \cdot 2 \mathrm{H}_{2} \mathrm{O}\right] \cdot \mathrm{H}_{2} \mathrm{O}\end{array}$} & $80(+)$ & $40-180$ & 10.6 & 10.7 & Dehydration \\
\hline & $260(-)$ & $180-280$ & 17.0 & 17.1 & Dehydrazination \\
\hline & $410(-)$ & $280-600$ & 85.1 & 85.2 & Decomposition to metal oxide \\
\hline \multirow{3}{*}{$\begin{array}{l}{\left[\mathrm{Co}\left\{3-\mathrm{C}_{6} \mathrm{H}_{4}\left(\mathrm{OCOCH}_{3}\right)\right.\right.} \\
\left.\mathrm{COO}\}_{2}\left(\mathrm{~N}_{2} \mathrm{H}_{4}\right) \cdot 2 \mathrm{H}_{2} \mathrm{O}\right] \cdot \mathrm{H}_{2} \mathrm{O}\end{array}$} & $80(+)$ & $60-160$ & 10.0 & 10.7 & Dehydration \\
\hline & $250(-)$ & $160-270$ & 17.0 & 17.1 & Dehydrazination \\
\hline & $450(-)$ & $270-600$ & 84.9 & 85.1 & Formation of metal oxide \\
\hline
\end{tabular}


Table 2. Continued.

\begin{tabular}{|c|c|c|c|c|c|}
\hline $\begin{array}{l}{\left[\mathrm{Cd}\left\{3-\mathrm{C}_{6} \mathrm{H}_{4}(\mathrm{OCOCH})_{3}\right)\right.} \\
\left.\mathrm{COO}\}_{2}\left(\mathrm{~N}_{2} \mathrm{H}_{4}\right) \cdot 2 \mathrm{H}_{2} \mathrm{O}\right] \cdot \mathrm{H}_{2} \mathrm{O}\end{array}$ & $\left.\begin{array}{c}80(+) \\
200(+) \\
240(-) \\
490(-)\end{array}\right\}$ & $\begin{array}{c}60-210 \\
210-320 \\
320-600\end{array}$ & $\begin{array}{c}9.9 \\
15.3 \\
77.1\end{array}$ & $\begin{array}{c}9.7 \\
15.4 \\
76.9\end{array}$ & $\begin{array}{l}\text { Dehydration } \\
\text { Dehydrazination } \\
\text { Formation of metal oxide }\end{array}$ \\
\hline $\begin{array}{l}{\left[\mathrm{Zn}\left\{3-\mathrm{C}_{6} \mathrm{H}_{4}\left(\mathrm{OCOCH}_{3}\right)\right.\right.} \\
\left.\mathrm{COO}\}_{2}\left(\mathrm{~N}_{2} \mathrm{H}_{4}\right) \cdot 2 \mathrm{H}_{2} \mathrm{O}\right] \cdot \mathrm{H}_{2} \mathrm{O}\end{array}$ & $\begin{array}{l}280(-) \\
440(-)\end{array}$ & $200-600$ & 84.1 & 84.0 & Formation of metal oxide \\
\hline $\begin{array}{l}{\left[\mathrm{Ni}\left\{4-\mathrm{C}_{6} \mathrm{H}_{4}\left(\mathrm{OCOCH}_{3}\right)\right.\right.} \\
\left.\mathrm{COO}\}_{2}\left(\mathrm{~N}_{2} \mathrm{H}_{4}\right)_{2}\right] \cdot 2 \mathrm{H}_{2} \mathrm{O}\end{array}$ & $\begin{array}{l}67(+) \\
281(-) \\
410(-)\end{array}$ & $\begin{array}{c}40-220 \\
220-250 \\
250-600\end{array}$ & $\begin{array}{c}6.4 \\
20.0 \\
85.5\end{array}$ & $\begin{array}{c}7.0 \\
19.3 \\
85.6\end{array}$ & $\begin{array}{l}\text { Dehydration } \\
\text { Dehydrazination } \\
\text { Formation of metal oxide }\end{array}$ \\
\hline $\begin{array}{l}{\left[\mathrm{Co}\left\{4-\mathrm{C}_{6} \mathrm{H}_{4}\left(\mathrm{OCOCH}_{3}\right)\right.\right.} \\
\left.\mathrm{COO}\}_{2}\left(\mathrm{~N}_{2} \mathrm{H}_{4}\right)_{2}\right] \cdot 2 \mathrm{H}_{2} \mathrm{O}\end{array}$ & $\left.\begin{array}{c}67(+) \\
210(-) \\
350(-) \\
400(-) \\
467(-)\end{array}\right\}$ & $\begin{array}{c}50-170 \\
170-250 \\
250-410 \\
410-600\end{array}$ & $\begin{array}{c}7.0 \\
19.18 \\
37.4 \\
85.7\end{array}$ & $\begin{array}{c}7.0 \\
19.3 \\
37.6 \\
85.5\end{array}$ & $\begin{array}{l}\text { Dehydration } \\
\text { Dehydrazination } \\
\text { Formation of metal phthalate } \\
\text { Formation of metal oxide }\end{array}$ \\
\hline $\begin{array}{l}{\left[\mathrm{Cd}\left\{4-\mathrm{C}_{6} \mathrm{H}_{4}\left(\mathrm{OCOCH}_{3}\right)\right.\right.} \\
\left.\mathrm{COO}\}_{2}\left(\mathrm{~N}_{2} \mathrm{H}_{4}\right)_{2}\right] \cdot 2 \mathrm{H}_{2} \mathrm{O}\end{array}$ & $\begin{array}{r}80(+) \\
250(-) \\
403(-)\end{array}$ & $\begin{array}{c}50-150 \\
150-320 \\
320-600\end{array}$ & $\begin{array}{c}6.0 \\
17.0 \\
78.0\end{array}$ & $\begin{array}{c}6.3 \\
17.5 \\
77.5\end{array}$ & $\begin{array}{l}\text { Dehydration } \\
\text { Dehydrazination } \\
\text { Formation of metal oxide }\end{array}$ \\
\hline $\begin{array}{l}{\left[\mathrm{Zn}\left\{4-\mathrm{C}_{6} \mathrm{H}_{4}\left(\mathrm{OCOCH}_{3}\right)\right.\right.} \\
\left.\mathrm{COO}\}_{2}\left(\mathrm{~N}_{2} \mathrm{H}_{4}\right)_{2}\right] \cdot 2 \mathrm{H}_{2} \mathrm{O}\end{array}$ & $271(-)$ & $200-600$ & 84.3 & 84.5 & Formation of metal oxide \\
\hline
\end{tabular}

Thermal data of $\left[\mathrm{Cd}\left(\mathrm{H}_{2} \mathrm{O}\right)_{3}\left(\left(\mathrm{C}_{6} \mathrm{H}_{4}(4-\mathrm{OH})(\mathrm{COO})\right)_{2}\right] \cdot \mathrm{H}_{2} \mathrm{O}\right.$

The TG curve showed a medium endothermic weight loss of $4 \%$ at $86{ }^{\circ} \mathrm{C}$ corresponding to elimination of one water molecule which might be of lattice type and another sharp endothermic weight loss of $12 \%$ at $220^{\circ} \mathrm{C}$ due to the elimination of three coordinated water molecules. The dehydration was immediately followed by a broad exotherm centered at $290{ }^{\circ} \mathrm{C}$ corresponding to oxidative decomposition showing a residual percentage of 27 for cadmium oxide.

\section{$X$-ray diffraction}

X-ray diffraction data of the complexes implies that the complexes with similar formulae showed isomorphism among themselves, which was evidenced from their ' $d$ ' spacings.

Crystal structure of $\left[\mathrm{Cd}\left(\mathrm{H}_{2} \mathrm{O}\right)_{3}\left(\left(\mathrm{C}_{6} \mathrm{H}_{4}(4-\mathrm{OH})(\mathrm{COO})\right)_{2}\right] \cdot \mathrm{H}_{2} \mathrm{O}\right.$

Crystal data of the above crystal are given in the Table 3. The significant bond lengths and bond angles of this crystal are listed in Table 4. Though Wang et al. [36] reported this crystal structure using 4-hydroxy benzoic acid as the starting material directly, this compound was obtained by the selective hydrolysis of 4-acetoxy benzoic acid to form 4-hydroxy benzoic acid which reacted with metal nitrate in this method.

The asymmetric unit of the crystal contains two molecules of the formula unit $\left(\mathrm{C}_{14} \mathrm{H}_{18} \mathrm{Cd}_{1} \mathrm{O}_{10}\right)$, which were present in the two different planes. Single crystal analysis revealed that the compound has a unique non-centro symmetric three dimensional frame work. The compound crystallized in the orthorhombic space $\mathrm{P}_{\mathrm{n}} 2_{1 \mathrm{a}}$ and there are two crystallographically similar complex units positioned in different planes. The polyhedron of the $\mathrm{Cd}$ atom $\left(\mathrm{Cd}_{2}\right)$ was a monocapped distorted octahedron formed by four oxygen atoms $(\mathrm{O} 7, \mathrm{O} 8, \mathrm{O} 10$ and $\mathrm{O} 11)$ from two bidentately chelating carboxylate ions of 4-hydroxy benzoate ligands, oxygen atom (O8W) of coordinated water and two oxygen atoms of another coordinated (O6W,O7W) water molecules taking apex positions with $\mathrm{Cd}-\mathrm{O}$ distances (carboxylato oxygen) varying between 2.3499-2.4028 $\AA$, Cd-O (water) distances varying between $2.2634(6 \mathrm{~W}), 2.3216(7 \mathrm{~W})$ and 2.3980 $\AA(8 \mathrm{~W})$. The lattice water $(\mathrm{O} 1 \mathrm{~W})$ was found to link with O9 via hydrogen bonding (H9A) and 
Table 3. Crystal data and structure refinement details of $\left[\mathrm{Cd}\left(\mathrm{H}_{2} \mathrm{O}\right)_{3}\left(\mathrm{C}_{6} \mathrm{H}_{4}(4-\mathrm{OH})(\mathrm{COO})\right)_{2}\right] \cdot \mathrm{H}_{2} \mathrm{O}$.

\begin{tabular}{|c|c|}
\hline \multicolumn{2}{|c|}{ Crystal data and structure refinement details } \\
\hline Empirical formula & $\mathrm{C}_{14} \mathrm{H}_{18} \mathrm{Cd}_{1} \mathrm{O}_{10}$ \\
\hline Formula weight & 458.690 \\
\hline Temperature (K) & 293 \\
\hline Wavelength $(\AA)$ & 0.71073 \\
\hline Crystal system & Orthorhombic \\
\hline Space group & $P_{\mathrm{n}} 2_{1 \mathrm{a}}$ \\
\hline \multicolumn{2}{|l|}{ Unit cell dimensions } \\
\hline $\mathrm{a}(\AA)$ & 10.3195 \\
\hline $\mathrm{b}(\AA)$ & 28.103 \\
\hline c $(\AA)$ & 12.0268 \\
\hline$\alpha\left(^{\circ}\right)$ & 90.00 \\
\hline$\beta\left(^{\circ}\right)$ & 90.00 \\
\hline$\gamma\left({ }^{\circ}\right)$ & 90.00 \\
\hline$v\left(\AA^{3}\right)$ & $3487.83(8)$ \\
\hline $\mathrm{Z}$ & 8 \\
\hline $\mathrm{D}_{\text {calcd }}\left(\mathrm{mg} \mathrm{m}^{-3}\right)$ & 1.7470 \\
\hline$\mu\left(\mathrm{mm}^{-1}\right)$ & 13.022 \\
\hline $\mathrm{F}(000)$ & 1840 \\
\hline Crystal size $\left(\mathrm{mm}^{3}\right)$ & $0.33 \times 0.32 \times 0.30$ \\
\hline$\theta$ range for data collection $\left(^{\circ}\right)$ & $2.50-27.00$ \\
\hline Limiting indeces & $-13,13 ;-34,37 ;-15,15$ \\
\hline Reflection collected unique & $7995 / 7308$ \\
\hline $\mathrm{R}_{\text {int }}$ & 0.0231 \\
\hline Absorption correlation & Empirical \\
\hline Refinement method & SHELXL 87 (Sheldrick 2008) \\
\hline No of parameters & 579 \\
\hline Goodness-of-fit on $\mathrm{F}^{2}$ & 1.118 \\
\hline Largest diff. Peak and hole $\left(\mathrm{e} \AA^{-3}\right)$ & 0.05960 .0314 \\
\hline
\end{tabular}

Table 4. Significant bond lengths $(\AA)$ and angles $\left({ }^{\circ}\right)$ of $\left[\mathrm{Cd}\left(\mathrm{H}_{2} \mathrm{O}\right)_{3}\left(\left(\mathrm{C}_{6} \mathrm{H}_{4}(4-\mathrm{OH})(\mathrm{COO})\right)_{2}\right] \cdot \mathrm{H}_{2} \mathrm{O}\right.$.

\begin{tabular}{|c|c|c|c|c|}
\hline \multicolumn{5}{|c|}{ Bond lengths and bond angles } \\
\hline $\mathrm{Cd} 2-\mathrm{O} 7$ & $2.3499(26)$ & \multicolumn{2}{|l|}{$\mathrm{Cd} 2-\mathrm{O} 8$} & $2.4028(25)$ \\
\hline $\mathrm{Cd} 2-\mathrm{O} 10$ & $2.3943(26)$ & \multicolumn{2}{|l|}{$\mathrm{Cd} 2-\mathrm{O} 11$} & $2.3679(21)$ \\
\hline $\mathrm{Cd} 2-\mathrm{O} 6 \mathrm{~W}$ & $2.2634(34)$ & \multicolumn{2}{|l|}{$\mathrm{Cd} 2-\mathrm{O} 7 \mathrm{~W}$} & $2.3216(30)$ \\
\hline $\mathrm{O} 1-\mathrm{Cd} 1-\mathrm{O} 5$ & $140.20(0.08)$ & \multicolumn{2}{|l|}{ O10-Cd2-O8W } & $80.84(0.09)$ \\
\hline $\mathrm{O} 2-\mathrm{Cd} 1-\mathrm{O} 4$ & $139.60(0.09)$ & \multicolumn{2}{|l|}{$\mathrm{O} 11-\mathrm{Cd} 2-\mathrm{O} 8 \mathrm{~W}$} & $135.33(0.09)$ \\
\hline $\mathrm{O} 2-\mathrm{Cd} 1-\mathrm{O} 5$ & $85.79(0.09)$ & \multicolumn{2}{|l|}{$\mathrm{O} 2-\mathrm{Cd} 1-\mathrm{O} 5 \mathrm{~W}$} & $88.37(0.10)$ \\
\hline O1- Cd1-O5W & $90.58(0.10)$ & \multicolumn{2}{|l|}{$\mathrm{O} 8-\mathrm{Cd} 2-\mathrm{O} 10$} & $141.58(0.09)$ \\
\hline $\mathrm{O} 7-\mathrm{Cd} 2-\mathrm{O} 10$ & $162.29(0.09)$ & \multicolumn{2}{|l|}{$\mathrm{O} 7-\mathrm{Cd} 2-\mathrm{O} 11$} & $41.43(0.08)$ \\
\hline Cd2-O7W-H7B & $103.59(3.86)$ & \multicolumn{2}{|l|}{ Cd2-O8W-H8B } & 109.49(3.86) \\
\hline Cd2-O7W-H7A & $114.69(3.25)$ & Cd2-O8W-H8A & & $115.73(3.38)$ \\
\hline \multicolumn{5}{|c|}{ Hydrogen bonding } \\
\hline D-H......A & D-H & H..A & D...A & D-H...A \\
\hline O6 W -H6A....O10(4) & $0.684(.040)$ & $2.077(.040)$ & $2.730(.004)$ & $160.17(4.45)$ \\
\hline O6 W -H6B ....O1 (0) & $0.716(.045)$ & $2.053(.045)$ & $2.758(.004)$ & $168.18(4.85)$ \\
\hline O7W -H7A..... & $0.754(.043)$ & $1.924(.042)$ & $2.663(.004)$ & $166.23(4.48)$ \\
\hline $\mathrm{O} 7 \mathrm{~W}-\mathrm{H} 7 \mathrm{~B} \ldots . . \mathrm{O} 6(6)$ & $0.782(.052)$ & $1.986(.052)$ & $2.765(.004)$ & $175.07(5.26)$ \\
\hline O8W -H8A....O11(3) & $0.901(.053)$ & $1.882(.052)$ & $2.780(.004)$ & $173.64(4.94)$ \\
\hline O8W -H8B ....O12(1) & $0.633(.042)$ & $2.233(.042)$ & $2.848(.004)$ & $164.51(4.81)$ \\
\hline
\end{tabular}


another lattice water $(\mathrm{O} 2 \mathrm{~W})$ was connected with $\mathrm{O} 6$ through hydrogen bonding $(\mathrm{H} 6 \mathrm{C})$ in a similar fashion. The planes containing any one carboxylic oxygen atoms (O8 and $\mathrm{O} 7)$ and (O10 and O11) bore an angle $141.5^{\circ}$. The hydrogen bond distance was found to vary between $1.882-$ $2.077 \AA$. The packing view of the crystal through axis 'a' is shown in Figure 4.

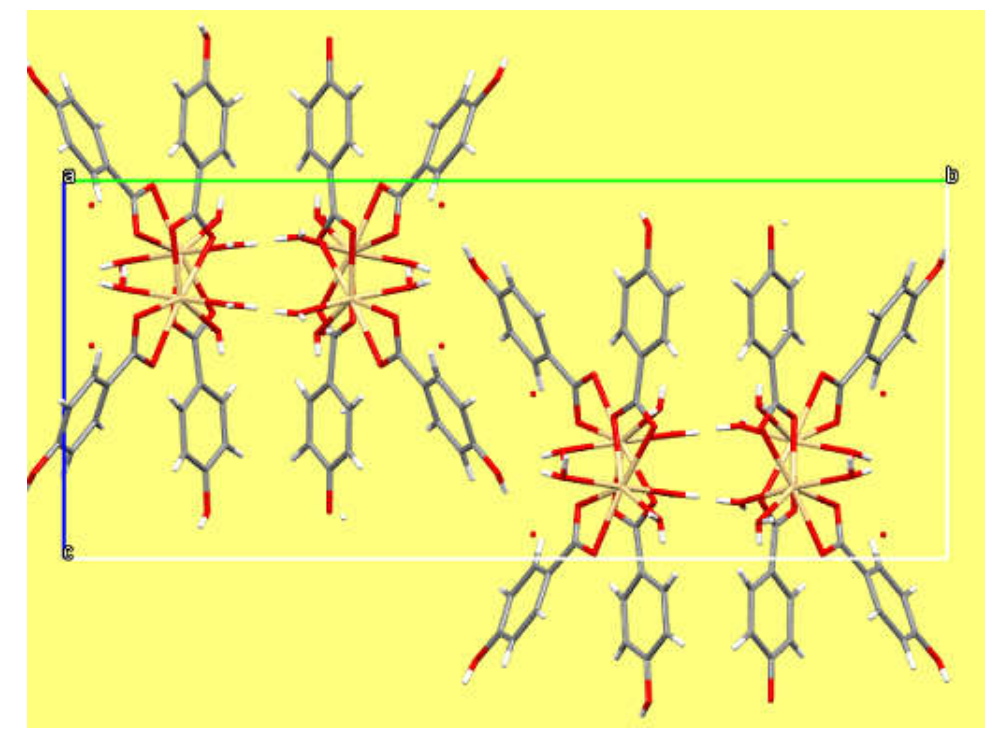

Figure 4. Packing view of $\left[\mathrm{Cd}\left(\mathrm{H}_{2} \mathrm{O}\right)_{3}\left(\mathrm{C}_{6} \mathrm{H}_{5}(4-\mathrm{OH}) \mathrm{COO}\right)_{2}\right] \cdot \mathrm{H}_{2} \mathrm{O}$ via axis 'a'.

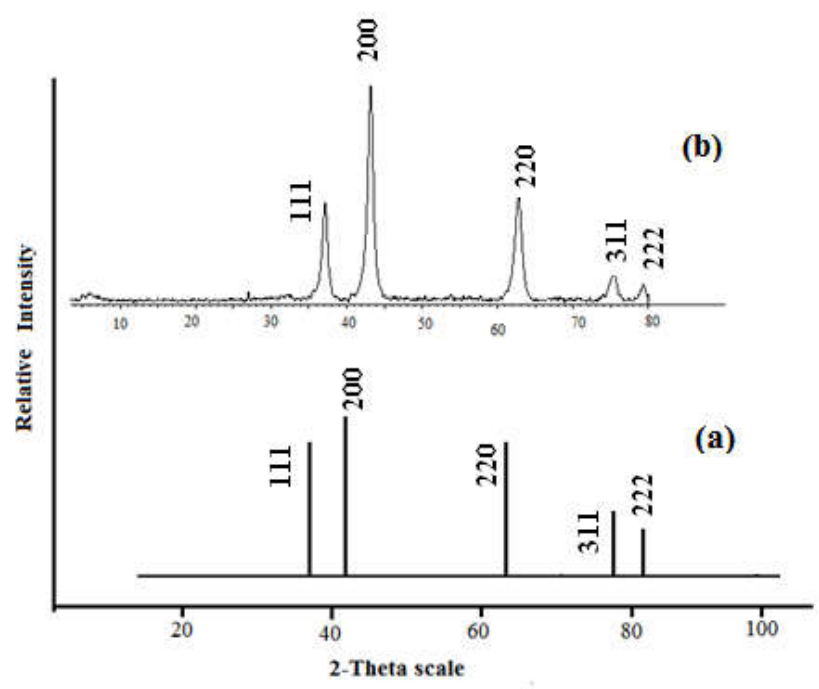

Figure 5. (a) Standard XRD pattern of $\mathrm{NiO}$ and (b) XRD pattern of final residue of $\left[\mathrm{Ni}\left\{3-\mathrm{C}_{6} \mathrm{H}_{4}\left(\mathrm{OCOCH}_{3}\right) \mathrm{COO}\right\}_{2}\left(\mathrm{~N}_{2} \mathrm{H}_{4}\right)\right] \cdot 3 \mathrm{H}_{2} \mathrm{O}$.

Bull. Chem. Soc. Ethiop. 2021, 35(2) 


\section{SEM-EDX studies}

The complexes were calcined in muffle furnace at their decomposition temperatures, kept at the same temperature for $10 \mathrm{~min}$. and then analyzed the cooled products for their morphology and particle size. The SEM-EDX images of residues obtained from $\left[\mathrm{Ni}(3-\mathrm{ab})_{2}\left(\mathrm{~N}_{2} \mathrm{H}_{4}\right)\right] \cdot 3 \mathrm{H}_{2} \mathrm{O}$ and the $\mathrm{XRD}$ patterns of the same $\mathrm{NiO}$ with the standard are shown in Figure 5. The metal oxide of $\left[\mathrm{Ni}(3-\mathrm{ab})_{2}\left(\mathrm{~N}_{2} \mathrm{H}_{4}\right)\right] \cdot 3 \mathrm{H}_{2} \mathrm{O}$ was found to be in nano scale of $18 \mathrm{~nm}$ as calculated from XRD using Scherer's formula [37] $\mathrm{D}=\mathrm{K} \lambda / \mathrm{B} \cos \theta$ where $\lambda$ is the $\mathrm{X}$-ray wavelength, $\mathrm{b}$ is the full width of height maximum (FWHM) of a diffraction peak, $\theta$ is the diffraction angle and $\mathrm{K}$ is the Scherer's constant of the order of 0.8 . Other two residues were micro sized metal oxides with irregular shapes.

\section{Conductance studies}

The molar conductance of selected complexes, $\left(\left[\mathrm{Zn}\left\{(2-\mathrm{ab})_{2}\left(\mathrm{~N}_{2} \mathrm{H}_{4}\right)\right\}\right] .3 \mathrm{H}_{2} \mathrm{O}, \quad\left[\mathrm{Co}\left\{(3-\mathrm{ab})_{2}\right.\right.\right.$ $\left.\left.\left(\mathrm{N}_{2} \mathrm{H}_{4}\right)\right\}\right] .3 \mathrm{H}_{2} \mathrm{O}$ and $\left[\mathrm{M}(4-\mathrm{ab})_{2}\left(\mathrm{~N}_{2} \mathrm{H}_{4}\right)_{2}\right] \cdot 2 \mathrm{H}_{2} \mathrm{O}$, where $\mathrm{M}=\mathrm{Ni}$ and $\left.\mathrm{Cd}\right)$ was determined using DMSO as solvent and compared with the reported values $[38,39]$. Except zinc and cadmium complexes (76 and $67 \mathrm{ohm}^{-1} \mathrm{~cm}^{2} \mathrm{~mol}^{-1}$, respectively), other transition metal complexes behaved as low conductivity electrolytes by showing molar conductance values in the range of 10-12 $\mathrm{ohm}^{-1} \mathrm{~cm}^{2} \mathrm{~mol}^{-1}$ indicating that they were neutral complexes. The high conductivity of zinc and cadmium complexes might be due to solvolysis by DMSO.

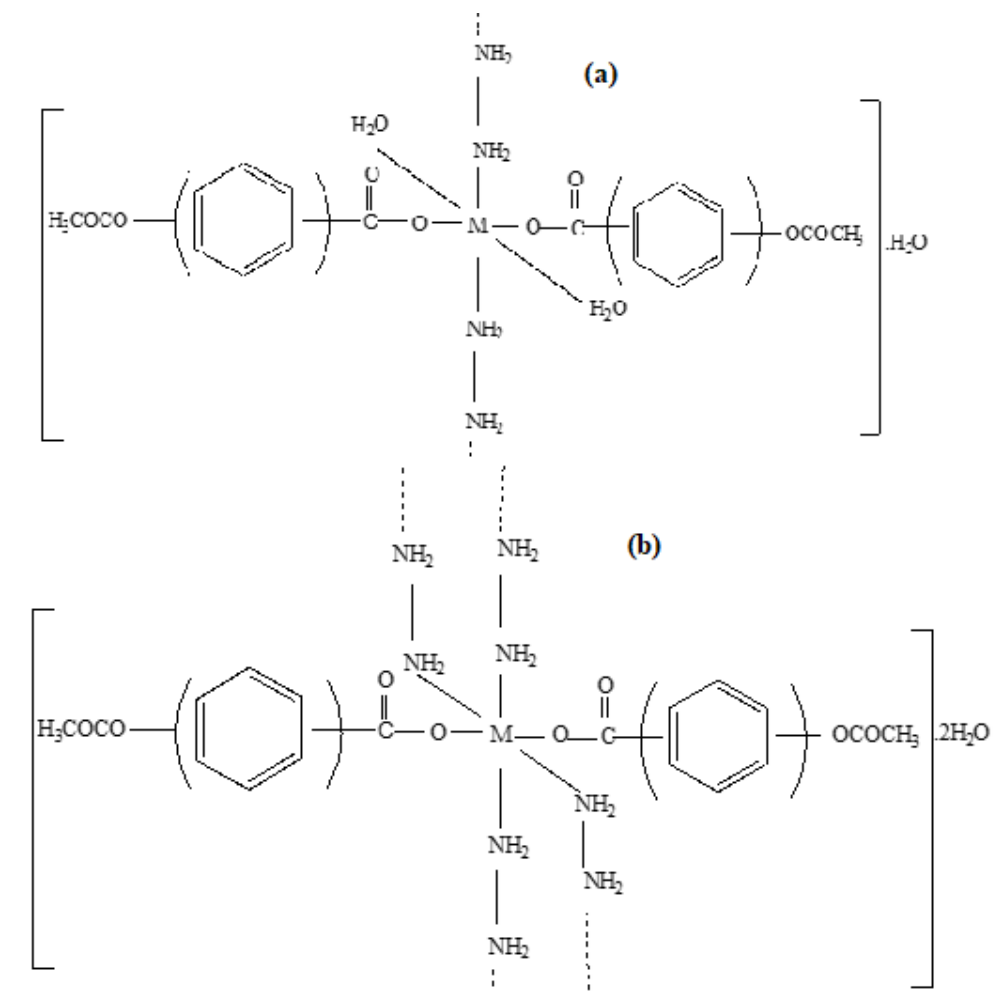

Figure 6. (a) Proposed structure of $\left[\mathrm{M}\left\{\left(2 \text { and 3) }-\mathrm{C}_{6} \mathrm{H}_{4}\left(\mathrm{OCOCH}_{3}\right) \mathrm{COO}\right\}_{2}\left(\mathrm{~N}_{2} \mathrm{H}_{4}\right) \cdot 2 \mathrm{H}_{2} \mathrm{O}\right] \cdot \mathrm{H}_{2} \mathrm{O}\right.$ and (b) $\left[\mathrm{M}\left\{4-\mathrm{C}_{6} \mathrm{H}_{4}\left(\mathrm{OCOCH}_{3}\right) \mathrm{COO}\right\}_{2}\left(\mathrm{~N}_{2} \mathrm{H}_{4}\right)_{2}\right] \cdot 2 \mathrm{H}_{2} \mathrm{O}$, where $\mathrm{M}=\mathrm{Ni}, \mathrm{Co}, \mathrm{Cd}$ and $\mathrm{Zn}$.

Bull. Chem. Soc. Ethiop. 2021, 35(2) 


\section{CONCLUSION}

The isomeric acetoxy benzoic acids such as 2-abH, 3-abH and 4-abH yielded the hydrazine complexes of transition metals having formula, $\left[\mathrm{M}(2-\mathrm{ab})_{2} \cdot\left(\mathrm{N}_{2} \mathrm{H}_{4}\right)\right] \cdot 3 \mathrm{H}_{2} \mathrm{O},\left[\mathrm{M}(3-\mathrm{ab})_{2} \cdot\left(\mathrm{N}_{2} \mathrm{H}_{4}\right)\right]$. $3 \mathrm{H}_{2} \mathrm{O}$ and $\left[\mathrm{M}(4-\mathrm{ab})_{2} \cdot\left(\mathrm{N}_{2} \mathrm{H}_{4}\right)_{2}\right] \cdot 2 \mathrm{H}_{2} \mathrm{O}$, where $\mathrm{M}=\mathrm{Ni}, \mathrm{Co}, \mathrm{Cd}$ and $\mathrm{Zn}$ on reaction with hydrazine hydrate and the respective metal ions. The acids involve in coordination with metal ions only through carboxyl groups and acetoxy group do not involve in coordination. Based on the studies, six coordination has been proposed for central metal ion with distorted octahedral geometry. However, the accurate structures can be known from single crystal XRD studies of the complexes. The proposed structures are given in Figure 6.

\section{Supplementary data}

Other crystallographic data have been deposited with the Cambridge Crystallographic Data Centre. Code number CCDC 800926. (Supplementary data is available on http:/CCDC.cam.ac.uk website).

\section{ACKNOWLEDGEMENTS}

The authors wish to acknowledge the (AICTE) All India Council for Technical Education, New Delhi, for sponsoring this study (grant in aid No./8023/BOR/RID/RPS-2, 2008-2009). They also wish to record their sincere thanks to Dr. M. Nethaji, Scientist, Department of Inorganic and Physical Chemistry, Indian Institute of Science, Bengaluru - 560012, Karnataka for his valuable help during single crystal X-ray diffraction analysis.

\section{REFERENCES}

1. Lawal, A.; Obaleye, J.A. Synthesis, characterization and antibacterial activity of aspirin and paracetamol metal complexes. Biokemistri 2007, 18, 9-15.

2. Ashraf, A.; Hanif, M.; Kubanik, M.; Sohnel, T.; Jamieson, S.M.F.; Bhattacharyya, A.; Hartinger, C.G. Aspirin-inspired organometallic compounds: Structural characterization and cytotoxicity. J.Organomet. Chem. 2017, 839, 31-37.

3. Ott, I.; Kircher.; B, Bagowski, C.P.; Vlecken, D.H.W.; Elisabeth, B.; Will, O.J.; Bensdorf, K.; Gust, R.; Sheldrick, W.S. Modulation of the biological properties of aspirin by formation of a bioorganometallic derivative. Angew. Chem. Int. Ed. 2009, 48, 1160-1163.

4. Rubner, G.; Bensdorf, K.; Wellner, A.; Kircher, B.; Bergemann, S.; Ott, I.; Gust, R. Synthesis and biological activities of transition metal complexes based on acetylsalicylic acid as neo-anticancer agents. J. Med. Chem. 2010, 53, 6889-6698.

5. Tarulli, S.; Quinzani, O.; Dristas, J.; Baram, E. Thermal behaviour of copper(II) complexes of haloaspirinates. J. Therm. Anal. Calorim. 2000, 60, 505-515.

6. Refat, M.S.; Sharshar, T.; Elsabawy, K.M.; Heiba, Z.K. Physicochemical impact studies of gamma rays on "aspirin" analgesics drug and its metal complexes in solid form: Synthesis, spectroscopic and biological assessment of $\mathrm{Ca}(\mathrm{II}), \mathrm{Mg}(\mathrm{II}), \mathrm{Sr}(\mathrm{II})$ and $\mathrm{Ba}(\mathrm{II})$ aspirinate complexes. J. Mol. Stru. 2013, 1047, 37-47.

7. Poyraz, M.; Banti, C.N.; Kourkoumelis, N.; Dokorou, V.; Manos, M.J.; Simcic, M.; Grdadolnik, S.G.; Mavromoustakos, T.; Giannoulis, A.D.; Verginadis, I.I.; Charalabopoulos, K.; Hadjikakou, S.K. Synthesis, structural characterization and biological studies of novel mixed ligand $\operatorname{Ag}(\mathrm{I})$ complexes with triphenylphosphine and aspirin or salicylic acid. Inorg. Chim. Acta 2011, 375, 114-121.

8. Ogodo, U.P.; Abosede, O.O. Synthesis and characterization of $\mathrm{Cu}(\mathrm{II})$ complexes of salicylate ligands. J. Appl. Sci. Environ. Manage. 2018, 22, 1961-1964. 
9. Kaur, H.; Dhir, K.; Kaur, J.; Mittu, B.; Chuhan, A. Synthesis and evaluation of diorganotin(IV) and triorganotin(IV) derivatives of aspirin, paracetamol and metronidazole as antimicrobial agents. Am. J. Drug Discov. Dev. 2013, 3, 13-22.

10. Ong, Y.C.; Blair, V.L.; Kedzierski, L.; Tuck, K.L.; Andrews, P.C. Stability and toxicity of tristolyl bismuth(V) dicarboxylates and their biological activity towards Leishmania major. Dalton Trans. 2015, 44, 18215-18226.

11. Islam, A.; Da Silva, J. G.; Berbet, F.M.; Da Silva, S M.; Rodrigues, B.L.; Beraldo, H.; Melo, M.N.; Frézard, F.; Demicheli, C. Novel triphenylantimony(V) and triphenylbismuth(V) complexes with benzoic acid derivatives: Structural characterization, in vitro antileishmanial and antibacterial activities and cytotoxicity against macrophages. Molecules 2014, 1, 60096030.

12. Ott, I.; Schmidt, K.; Kircher, B.; Schumacher, P.; Wiglenda, T.; Gust, R. Antitumor-active cobalt-alkyne complexes derived from acetylsalicylic acid: Studies on the mode of drug action. J. Med. Chem. 2005, 48, 622-629.

13. Mandal, P.; Kundu, B.K.; Vidya Sabu, K.V.; Helen, A.; Dhankhar, S.S.; Nagaraja, C.M.; Bhattacherjee, D.; Bhabak, K.P.; Mukhopadhyay. S. Ruthenium(II) arene NSAID complexes: Inhibition of cyclooxygenase and antiproliferative activity against cancer cell lines. Dalton Trans. 2018, 47, 517-527.

14. Aziz, A.A.; S.A. Raoof, S.A.; Gameel Hasanb, W.M.; Saiedb, S.M. Preparation of new complexes from a mixture of aspirin (acetylsalicylic acid), paracetamol and methyldopa with divalent manganese, iron, cobalt, nickel and copper, with a study of their physical and chemical properties. Egypt. J. Chem. 2021, 64, 2405-2413.

15. Mizerovskii, L.N.; Khanzutina, L.V.; Kuznetsov, A.K. Thermodynamics of the reaction of copolycondensation of 4-acetoxybenzoic and 6-acetoxy-2-naphthoic acids. Fibre Chem. 1997, 29, 292-297.

16. Williams, P.A.; Han, X.; Padias, A.B.; Hall, Jr. H.K. Kinetics of the polymerization of 4acetoxybenzoic acid and 6-acetoxy-2-naphthoic acid. Macromolecules 1996, 29, 1874-1879.

17. Manuel Monte, J.S.; Luis Santos, M.N.B.F.; Fonseca, J.M.S.; Sousa C.A.D. Vapour pressures, enthalpies and entropies of sublimation of para substituted benzoic acids. $J$. Therm.. Anal. Calorim. 2010, 100, 465-474.

18. Kang, T.K.; Kim, Y.; Ha, C.S. Rheological and thermal properties of blends of modified poly(ethylene terephthalate) with $p$-acetoxybenzoic acid and poly(butylene terephthalate). $J$. Appl. Polym. Sci. 1999, 74, 1797-1806.

19. Bishara, A.; Al-Mulla, A.; Al-Roomi, Y.; Shaban, H. Synthesis of liquid crystalline polymers from polyethylene terephthalate and 4-acetoxybenzoic acid: A kinetic study. $J$. Appl. Polym. Sci. 2012, 123, 1359-1369.

20. Chipley, J.R.; Uraih, N. Inhibition of Aspergillus growth and aflatoxin release by derivatives of benzoic acid. Appl. Environ. Microbiol. 1980, 40, 352-357.

21. Arunadevi, N.; Vairam, S. 3-Hydroxy-2-naphthoate complexes of transition metals with hydrazine - preparation, spectroscopic and thermal studies. E. J. Chem. 2009, 6(S1), S413S421.

22. Arunadevi, N.; Vairam, S. Thermal and spectroscopic studies of divalent transition metal hydrazine complexes with 2-naphthoic acetic acid. J. Eng. Comput. Appl. Sci. 2012, 1, 1-8.

23. Manimekalai, R.; Sinduja, C.R.; Kalpanadevi, K. Coordination aspects of newly synthesised complexes of some divalent transition metals with 2,4-dichlorophenoxy acetate and hydrazine. Chem. Sci. Trans. 2013, 2, 1004-1008.

24. Helen Pricilla Bai, E.; Vairam, S. Transition metal complexes of acetamido benzoic acids with hydrazine - Spectral and thermal studies. Asian. J. Chem. 2013, 25, 209-216.

25. Mohanapriya, S.; Muthukumaran, C.; Vairam, S. Synthesis, characterization, thermal behavior and antimicrobial activity of 3-methyl benzoate complexes of transition metal with hydrazine. Bull. Chem. Soc. Ethiop. 2016, 30, 241-252. 
26. Kuppusamy K.; Govindarajan, S. Benzoate complexes of dipositive first row transition metal ions with hydrazine. Synth. React. Inorg. Met. Org. Chem. 1996, 26, 225-243.

27. Yasodhai, S.; Govindarajan, S. Coordination compounds of some divalent metals with hydrazine and dicarboxylate bridges. Synth. React. Inorg Met.Org. Chem. 2000, 30, 745760.

28. Vairam, S.; Premkumar, T.S.; Govindarajan, S. Trimellitate complexes of divalent transition metals with hydrazinium cation. J. Therm. Anal.Calorim. 2010, 100, 955-960.

29. Vairam, S.; Premkumar, T.S.; Govindarajan, S. Preparation and thermal behavior of divalent transition metal complexes of pyromellitic acid with hydrazine. J Therm. Anal. Calorim. 2010, 101, 979-985.

30. Helen Pricilla Bai, E.; Vairam, S. Hydrazine complexes of lanthanides with 3-acetoxy- and 4-acetoxybenzoic acids: spectroscopic, thermal, and XRD studies. J. Chem. 2013, 1-10. https://doi.org/10.1155/2013/717618.

31. Vogel, I.A. A Text Book of Quantitative Inorganic Analysis, Longmans: London; 1975; pp. 380 and 433.

32. Lever, A.B.P. Inorganic Electronic spectroscopy, Elsevier: Amsterdam; 1984.

33. Nakamoto, K. A Text Book of Infrared and Raman Spectra of Inorganic and Coordination compounds, John Wiley and Sons: New York; 1986.

34. Vandenbelt, J.M. Properties and behavior of the acetoxy group in ultraviolet absorption spectroscopy. Appl. Spectrosc. 1963, 17, 120-121.

35. Cardarelli, E.; D’Ascenzo, G.; Magri Pupella, A.D. Complexes of cobalt(II), nickel(II) and copper(II) with the benzenedicarboxylic acids. Thermal properties. Thermochim. Acta 1979, 33, 267-273.

36. Wang, R.H.; Hong, M.C.; Chen, J.T.; Weng, J.B.; Cao, R.; Zhao, Y.J. Synthesis and crystal structure of a seven-coordinate cadmium(II) complex. Chinese J. Strut. Chem. 2002, 21, 268-272.

37. Cao, G. Nano Structures and Nano materials, Synthesis, Properties and Applications, Imperial College Press: London; 2004.

38. Geary, W.J. The use of conductivity measurements in organic solvents for the characterization of coordination compounds. Coord. Chem. Rev. 1971, 7, 81-122.

39. Gull, P.; Talukdar, Md. I.A.; Dar, O.A.; Malik, M.A.; Hashmi, A.A. Synthesis, spectroscopic characterization, coordination, and antimicrobial activity of some metal complexes derived from 1,2-diphenylethane-1,2-dione and dinitrophenyl hydrazine Schiff base ligand. Jundishapur J. Nat. Pharm. Prod. 2018, 13, e67179. DOI: 10.5812/jjnpp.67179. 